\title{
Analysis and Optimisation of Wind-Induced Vibration Control for High-Rise Chimney Structures
}

\author{
Zhao-Dong Xu, Jun-Tao Zhu and Deng-Xiang Wang \\ Key Laboratory of C\&PC Structures of the Ministry of Education, Southeast University, Nanjing, China
}

\begin{abstract}
(Received 7 September 2012; provisionally accepted 15 November 2012; accepted 13 February 2013)
Wind is a key factor when determining the safety of high-rise structures, such as buildings, chimneys, or towers. Using dampers to control wind-induced vibration is a safe, effective, and economical method for high-rise structures to employ. In this paper, viscoelastic dampers (VEDs) were used to reduce the dynamic responses of a 75-metre-high chimney. First, a simulation method for the stochastic wind field, based on the modified Fourier spectrum, was proposed. The method provided the accurate data of the wind velocity time history, which then simulated wind pressure through the use of a numerical wind tunnel. Then, the finite element model for the Madagascar chimney structure was built, and a wind-induced vibration analysis of the structure with and without VEDs was carried out under the simulated wind excitation. The optimisation - method, based on the genetic algorithm, was used to optimise the location of the VEDs. It was concluded that the accuracy of the modified Fourier spectrum method (MFSM) was greatly improved, when compared to the spectrum representation method of simulating the stochastic wind field. VEDs can effectively reduce the dynamic responses of chimney towers, especially for the displacement responses. In addition, the proposed optimisation method quickly determined the optimum positions and necessary quantities of VEDs to use, which yielded effective vibration mitigation.
\end{abstract}

\section{INTRODUCTION}

With the advancement of construction techniques, civil engineering structures have become larger, higher, and lighter. Some examples of such structures include high-rise buildings, skyscrapers, long-span space structures, and bridges. All such modern structures are extremely sensitive to wind excitation. As is well known, wind is a natural force with great destructive power. Damage due to excessive wind has become a serious problem lately, due to global warming, and has led to a loss of tens or hundreds of billion dollars per year to the global economy. Therefore, it is significant to study wind vibration responses and to take reasonable control measures when constructing high-rise structures.

Making accurate predictions of wind loads and their effects on high-rise structures is therefore a necessary step in the design process, and static analysis under wind loads is not suitable for structural design and the corresponding safety targets. Therefore, dynamic analysis of these structures under wind loads is becoming more and more significant. A time-domain analysis method is usually applied in dynamic analysis under wind loads, which provides evidence for structural design and fatigue analysis, but wind velocity time history around structures should be determined before time-domain dynamic analysis is tested. Subsequently, the wind velocity time history should be changed into wind pressure time history based on the wind pressure distribution characteristics on the structural surface. ${ }^{1-3}$ Generally, wind velocity time history is generated according to wind velocity spectrums with special characteristics, such as the Davenport Spectrum, the Kaimal Spectrum, and the Harris Spectrum, which are summarized based on strong wind records. Such wind velocity spectrums are highly representative and accurate, which satisfy the demands of simulation of wind velocity time history and dynamic anal- ysis. However, field measurements are not economic and lead to heavy workload, which limits wider application. Currently, the spectrum representation method ${ }^{4}$ and the autoregressive method $^{5}$ are usually applied in the simulation of the stochastic wind field. The autoregressive method is based on a linear filtering technique: i.e., a white noise process with an average value of zero is passed through a filter, and a random process with a special spectrum is exported. The autoregressive method is efficient and requires little calculation, which leads to fast calculation speed. However, this method is less accurate when compared to the spectrum representation method. In fact, the precision of the spectrum representation method usually does not meet the calculation requirements. Moreover, the spectrum representation method has the drawback of a slow calculation speed. Therefore, the modified Fourier spectrum method (MFSM) has been introduced into the simulation of a stochastic wind field for the purpose of this paper. The phase information of a simulated point was determined by the cross power spectral density (CPSD) between the simulation and the adjacent points. Combined with the auto power spectral density (APSD) of the simulation points, an iterative modification for the Fourier spectrum was carried out. The wind velocity time history, with special time and space correlation, was then generated. Finally, Simulation of the wind field for a highrise chimney was undertaken, to verify the effectiveness of the MFSM.

Another important point of research regards the process of taking measures to reduce the wind-induced vibration responses of high-rise structures. In 1972, $\mathrm{Yao}^{6}$ first proposed the concept of structural control. The viscoelastic damper (VED) is the common passive control method. The main advantages of employing VEDs are to mitigate structural vibration most economically and effectively, while also achiev- 